\title{
A SIMPLE PROOF OF THE CLASSIFICATION OF RATIONAL ROTATION $C^{*}$-ALGEBRAS
}

\author{
HONG-SHENG YIN
}

\begin{abstract}
A simple proof of the classification of rational rotation $C^{*}$ algebras is given.
\end{abstract}

In recent years the irrational and rational rotation $C^{*}$-algebras have attracted much attention. The irrational rotation $C^{*}$-algebras were classified by combining the work of Rieffel [7] and Pimsner and Voiculescu [6]. The rational rotation $C^{*}$ algebras were first classified by [4], then different proofs were given in $[8,2,1$ and 5]. It is remarkable that all of the five proofs in the rational case are quite different from the proof in the irrational case. In this note we will show that the powerful methods in [6 and 7$]$ work as well in the rational case and thus give a very simple proof (the sixth) of the classification of rational rotation $C^{*}$-algebras. Our proof is based on the following two observations.

First, the irrational rotation $C^{*}$-algebras each have a unique tracial state, but the rational rotation $C^{*}$-algebras have infinitely many tracial states. This seems to be an obstruction to using the methods of [6 and 7]. But Elliott [3] has already shown that at the $K_{0}$-level all of these tracial states give the same map from the $K_{0}$-group to $\mathbf{R}$. Second, let $A$ and $B$ be two unital $C^{*}$-algebras such that all of their tracial states give the same map from their $K_{0}$-groups to $\mathbf{R}$. Then any isomorphism $\phi: A \rightarrow B$ would induce a commutative diagram

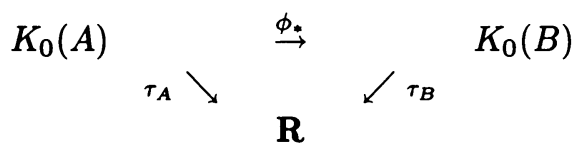

where $\tau_{A}$ and $\tau_{B}$ are induced from tracial states on $A$ and $B$, respectively. The classification of irrational rotation $C^{*}$-algebras is obtained by comparing the images of their $K_{0}$-groups under the induced trace maps. But that method does not work in the rational case. What we really need is the above commutative diagram, which contains much more information than the equality $\tau_{A}\left(K_{0}(A)\right)=\tau_{B}\left(K_{0}(B)\right)$.

Now we start to classify the rotation $C^{*}$-algebras $A_{\theta}=C\left(S^{1}\right) \times_{\theta} \mathrm{Z}$, where the action $\theta$ on $C\left(S^{1}\right)$ is given by a rotation of angle $2 \pi \theta$ on the unit circle $S^{1}$, and $0 \leq \theta<1$. In the following theorem and its proof, we deal with all $A_{\theta}$ 's at the same time. But the proof for the irrational case is exactly the same as the original one given in [6 and 7 ].

THEOREM. $A_{\theta_{1}}$ is isomorphic to $A_{\theta_{2}}$ iff $\theta_{1}=\theta_{2}$ or $\theta_{1}=1-\theta_{2}$.

ProOF. By [7 and 6], $K_{0}\left(A_{\theta}\right) \simeq Z^{2}$ with generators [1] and [p], where $p$ is a projection in $A_{\theta}$ such that if $\tau_{A_{\theta}}$ is the canonical trace on $A_{\theta}$ then $\tau_{A_{\theta}}(p)=\theta$. The

Received by the editors October 16, 1985.

1980 Mathematics Subject Classification. Primary 46L35; Secondary 46M20. 
proofs given in [7 and 6] are for irrational $\theta$, but they work as well for rational $\theta \neq 0$ as is easily checked. When $\theta=0, p$ should be taken as the Bott projection in $A_{\theta} \otimes M_{2}$, but this does not cause any difficulty in the classification since $A_{0}$ is the only $C^{*}$-algebra among all $A_{\theta}$ 's with $\tau_{A_{\theta}}\left(K_{0}\left(A_{\theta}\right)\right) \subset Z$. Let $\phi: A_{\theta_{1}} \rightarrow A_{\theta_{2}}$ be an isomorphism. By [3], all tracial states of $A_{\theta}$ give the same map from $K_{0}\left(A_{\theta}\right)$ to R. Hence we get a commutative diagram

$$
Z \oplus Z\left[p_{1}\right] \simeq K_{0}\left(A_{\theta_{1}}\right){ }_{\tau_{A_{\theta_{1}}} \searrow} \underbrace{\stackrel{\phi_{*}}{\rightarrow}}_{\mathbf{R}} K^{\swarrow \tau_{A_{\theta_{2}}}} K_{0}\left(A_{\theta_{2}}\right) \simeq Z \oplus Z\left[p_{2}\right]
$$

Since $\phi_{*}([1])=[1]$ and $\phi_{*} \in G L(2, \mathbf{Z}), \phi_{*}$ must be of the form $\left(\begin{array}{ll}1 & n \\ 0 & \pm 1\end{array}\right)$. The commutativity of the above diagram then gives

$$
\begin{aligned}
\theta_{1} & =\tau_{A_{\theta_{1}}}\left(p_{1}\right)=\tau_{A_{\theta_{1}}}\left(\left[p_{1}\right]\right)=\tau_{A_{\theta_{2}}}\left(\phi_{*}\left(\left[p_{1}\right]\right)\right) \\
& =\tau_{A_{\theta_{2}}}\left(n[1] \pm\left[p_{2}\right]\right)=\tau_{A_{\theta_{2}}}(n) \pm \tau_{A_{\theta_{2}}}\left(p_{2}\right)=n \pm \theta_{2} .
\end{aligned}
$$

Since $0 \leq \theta<1$, we conclude that $\theta_{1}=\theta_{2}$ or $\theta_{1}=1-\theta_{2}$.

The converse of the theorem is obvious. Q.E.D.

ACKNOWLEDGEMENT. I would like to thank my supervisor Professor John Phillips and my temporary supervisor Professor Peter Fillmore for their careful reading of and comments on this paper. This research was supported by a Killam Scholarship from Dalhousie University.

\section{REFERENCES}

1. M. D. Brabanter, The classification of rational rotation $C^{*}$-algebras, Arch. Math. (Basel) 43 (1984), 79-83.

2. S. Disney and I. Raeburn, Homogeneous $C^{*}$-algebras whose spectra are tori, J. Austral. Math. Soc. Ser. A 38 (1985), 9-39.

3. G. A. Elliott, On the $K$-theory of the $C^{*}$-algebra generated by a projective representation of a torsion-free discrete abelian group, Operator Algebras and Group Representations, Vol. I, Pitman, 1983, pp. 157-184.

4. R. Hoegh-Krohn and T. Skjelbred, Classification of $C^{*}$-algebras admitting ergodic actions of the two-dimensional torus, J. Reine Angew. Math. 328 (1981), 1-8.

5. J. A. Packer, $C^{*}$-algebras generated by projective representations of the discrete Heisenberg group. I, preprint.

6. M. Pimsner and D. Voiculescu, Exact sequences for K-Groups and Ext-groups of certain crossed products, J. Operator Theory 4 (1980), 93-118.

7. M. Rieffel, $C^{*}$-algebras associated with irrational rotations, Pacific J. Math. 93 (1981), 415-429.

8. _ The cancellation theorem for projective modules over irrational rotation $C^{*}$ algebras, Proc. London Math. Soc. 47 (1983), 285-302.

Department of Mathematics, Dalhousie University, Halifax, Nova Scotia, CANADA, B3H $4 \mathrm{H} 8$

Current address: Department of Mathematics, University of Ottawa, Ottawa, Ontario, Canada KIN 6N5 